\title{
Identification of candidates for cyclotide biosynthesis and cyclisation by expressed sequence tag analysis of Oldenlandia affinis
}

\author{
Qiaoping Qin ${ }^{1,2}$, Emily J McCallum ${ }^{1,5}$, Quentin Kaas ${ }^{1}$, Jan Suda ${ }^{3,4}$, Ivana Saska ${ }^{1,6}$, David J Craik1, Joshua S Mylne ${ }^{1 *}$
}

\begin{abstract}
Background: Cyclotides are a family of circular peptides that exhibit a range of biological activities, including antibacterial, cytotoxic, anti-HIV activities, and are proposed to function in plant defence. Their high stability has motivated their development as scaffolds for the stabilisation of peptide drugs. Oldenlandia affinis is a member of the Rubiaceae (coffee) family from which 18 cyclotides have been sequenced to date, but the details of their processing from precursor proteins have only begun to be elucidated. To increase the speed at which genes involved in cyclotide biosynthesis and processing are being discovered, an expressed sequence tag (EST) project was initiated to survey the transcript profile of $O$. affinis and to propose some future directions of research on in vivo protein cyclisation.
\end{abstract}

Results: Using flow cytometry the holoploid genome size (1C-value) of O. affinis was estimated to be 4,210 - 4,284 Mbp, one of the largest genomes of the Rubiaceae family. High-quality ESTs were identified, 1,117 in total, from leaf cDNAs and assembled into 502 contigs, comprising 202 consensus sequences and 300 singletons. ESTs encoding the cyclotide precursors for kalata B1 (Oak1) and kalata B2 (Oak4) were among the 20 most abundant ESTs. In total, 31 ESTs encoded cyclotide precursors, representing a distinct commitment of $2.8 \%$ of the 0 . affinis transcriptome to cyclotide biosynthesis. The high expression levels of cyclotide precursor transcripts are consistent with the abundance of mature cyclic peptides in O. affinis. A new cyclotide precursor named Oak5 was isolated and represents the first CDNA for the bracelet class of cyclotides in $O$. affinis. Clones encoding enzymes potentially involved in processing cyclotides were also identified and include enzymes involved in oxidative folding and proteolytic processing.

Conclusion: The EST library generated in this study provides a valuable resource for the study of the cyclisation of plant peptides. Further analysis of the candidates for cyclotide processing discovered in this work will increase our understanding and aid in reconstructing cyclotide production using transgenic systems and will benefit their development in pharmaceutical applications and insect-resistant crop plants.

\section{Background}

The first cyclotide was isolated from the plant Oldenlandia affinis, a member of the Rubiaceae (coffee) family, which is widely distributed throughout the Congo region of Africa. Pregnant women of the Lulua tribe would drink a tea made from an infusion of $O$. affinis leaves in order to accelerate their labours. In the early 1970s, these uterotonic properties were attributed to a peptide named kalata B1 $[1,2]$. It was not until 20 years later

\footnotetext{
* Correspondence: j.mylne@imb.uq.edu.au

${ }^{1}$ The University of Queensland, Institute for Molecular Bioscience, Brisbane, Queensland, 4072, Australia
}

that the structure of kalata B1 was described as having a backbone that is head-to-tail cyclised and possessing a disulfide knotted three-dimensional structure [3]. Kalata B1 was later shown to be produced from a larger precursor protein [4]. The name "cyclotide" was coined [5] to describe peptides with similar properties in the Rubiaceae, Violaceae and Cucurbitaceae families. With over 100 cyclotides already sequenced, a dozen structures determined and thousands more predicted from mass spectrometry profiles, cyclotides could be the largest known family of plant peptides [6]. 
Cyclotides are exceptionally resistant to thermal and biochemical extremes and to treatment with endoproteases [7]. It is thought that cyclotides function as defence molecules within plants, as some have been shown to have insecticidal properties $[4,8,9]$. This stability has motivated the development of cyclotides as scaffolds for the stabilisation and presentation of bioactive peptides in drug design $[10,11]$. They also have a range of potential applications in agriculture, given their insecticidal properties [8] and nematocidal activities [12-14].

O. affinis is a perennial herb with a woody root that grows at altitudes up to 1,500 metres above sea level [15]. Eighteen $O$. affinis cyclotides have been sequenced to date $[16,17]$, but their processing mechanisms have only begun to be elucidated $[18,19]$. Cyclotides are notably absent in model plants Arabidopsis thaliana and Nicotiana tabacum (tobacco). Transgenic expression of precursor proteins for cyclotide kalata B1 in Arabidopsis and tobacco produces a small proportion of the correctly processed cyclic form in addition to a number of mis-processed (truncated or elongated) linear forms not usually found in $O$. affinis $[18,19]$. This observation suggests that plants which do not produce cyclotides still contain machinery capable of processing them. However, efficient processing might require specially adapted pathways. Alternatively, these species might contain additional pathways that degrade cyclotides or inhibit proper cyclotide processing.

Sequencing of expressed sequence tags (ESTs) obtained from plant tissues and organs has been used to provide insights into the machinery underpinning biological processes. Lange et al. [20] found enzymes responsible for essential oil metabolism using ESTs from a cDNA library made from Mentha $\times$ piperita (peppermint) leaves rich in essential oils. Davis et al. [21] later used ESTs to elucidate the nature of production of menthone, the predominant monoterpene produced in the essential oil of maturing peppermint. Using an EST approach, Newcomb et al. [22] discovered genes responsible for fruit ripening, flavour, colour and biosynthesis of health-related compounds in apple.

In this study, we estimated the genome size of $O$. affinis using flow cytometry and report an EST sequencing project for $O$. affinis that resulted in the isolation of 1,117 high-quality ESTs. This is the first significant sequencing effort of $O$. affinis and a step towards building a valuable resource that will help understand the molecular basis of cyclotide biosynthesis. We found $O$. affinis commits $2.8 \%$ of its transcriptome to cyclotide precursors and $2.4 \%$ to proteins predicted to be involved in cyclotide processing. Discovery of the proteins responsible for efficient processing of cyclic peptides will enable the efficient use of model plants or plant cells for generating cyclotides or engineered molecules with pharmaceutically active peptides grafted within the cyclic peptide backbone. These two possibilities could lead to novel crop protection strategies and the use of plants as tools for cost-effective production of peptide drugs.

\section{Results and Discussion \\ Oldenlandia affinis genome size}

The somatic chromosome number of $O$. affinis is $2 \mathrm{n}=$ $18(\mathrm{x}=9)$, which is in agreement with other Oldenlandia species, such as O. corymbosa, where chromosome numbers are nine or a multiple thereof [15]. The genome size of $O$. affinis was determined by using flow cytometry with Glycine max 'Polanka' $(2 \mathrm{C}=2.50 \mathrm{pg})$ as an internal reference standard. Figure 1 shows the DNA histogram obtained from the $O$. affinis seedlings and the internal reference standard. Genome sizes (mean 2Cvalues $\pm \mathrm{SD}$ ) for $O$. affinis seedlings and adult plants were estimated to be $8.61 \pm 0.08 \mathrm{pg}$ and $8.76 \pm 0.07 \mathrm{pg}$, respectively. Considering $1 \mathrm{pg}=0.978 \times 10^{9} \mathrm{bp}=978$ Mbp [23], the monoploid genome size is between 4,210 and 4,284 Mbp. Certain variations between different ontogenetic stages (difference 1.7\%) can be due to different levels of secondary metabolites and instrument fluctuation. $1 C$-values in Rubiaceae plants vary from 0.35 to $5.08 \mathrm{pg}$, with median and mean values of $0.83 \mathrm{pg}$ and $1.11 \mathrm{pg}$, respectively http://data.kew.org/cvalues. O. affinis therefore possesses one of the largest holoploid genomes of the Rubiaceae family.

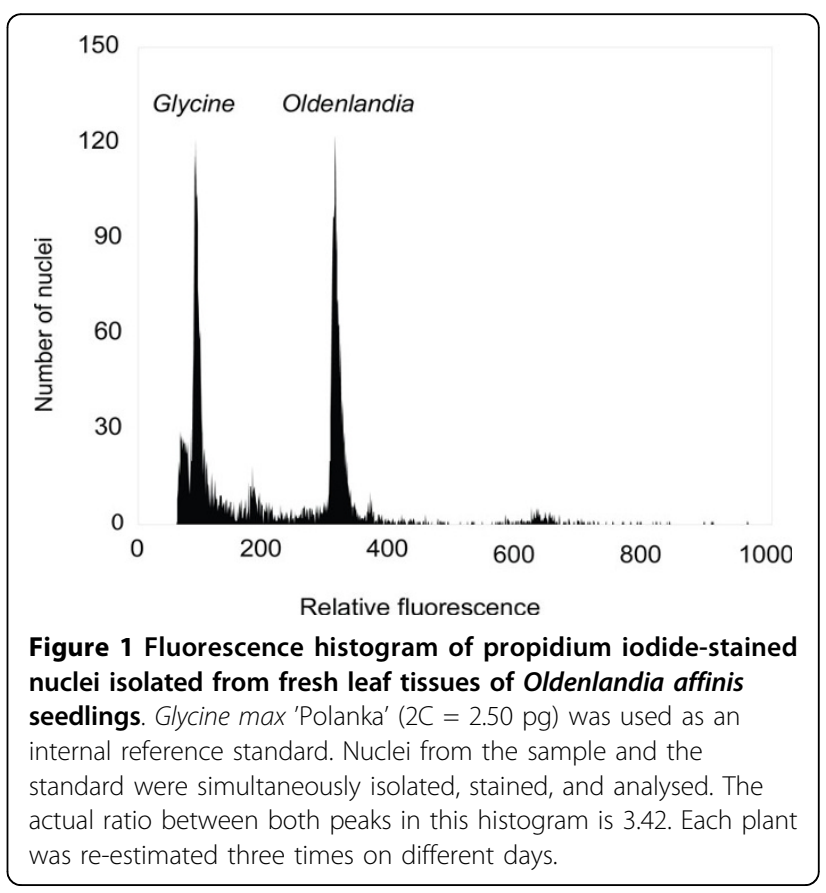




\section{EST sequencing and analysis}

Using a cDNA library made from O. affinis leaves [4], we generated 1,163 ESTs. After editing raw sequences for quality and discarding sequences less than $100 \mathrm{bp}$ in length, 1,117 sequences remained with an average read length of $624 \mathrm{bp} .60 \%$ of the ESTs were above $600 \mathrm{bp}$ and $48 \%$ were above 700 bp (Table 1). All edited EST sequences were submitted to GenBank dbEST [GenBank: GE470292-GE471408]. The 1,117 ESTs were clustered into 502 contigs, comprising 202 consensus sequences and 300 singletons using SeqMan sequence assembly software (DNASTAR). Clone redundancy was $45 \%$. Of the total ESTs, $27 \%$ represent singletons, which is similar to the values of $27 \%$ in cotton [24], $27.1 \%$ in bean [25], and $29 \%$ in pineapple [26]. To investigate the level of redundancy, contigs were listed by clone abundance, from contigs containing the largest number of ESTs to those containing a single EST. Almost half of the ESTs (48\%) were found to cluster within just $16 \%$ of contigs, thus transcription of a small subset of genes appears to dominate a high proportion of the transcriptome. However, as library construction involved an amplification step, the possibility that amplification bias might have contributed to the high level of redundancy cannot be discounted.

\section{Bioinformatics}

To assign putative functions to O. affinis ESTs, sequences were exported in FASTA format and conducted with the Basic Local Alignment Search Tool (BLASTX) and performed against the GenBank nonredundant database $[27,28]$. A stringent BLASTX cut-off of $10 \mathrm{E}^{-20}$ was chosen to ensure annotations were based on cDNAs with a high degree of similarity. Using these

\section{Table 1 Summary of ESTs from Oldenlandia affinis leaf} cDNA library

\begin{tabular}{lcccc}
\hline & Length & No. ESTs & $\%$ & Cumulative \% \\
\hline Minimum sequence length & $105 \mathrm{bp}$ & & & \\
Average sequence length & $624 \mathrm{bp}$ & & & \\
Maximum sequence length & $926 \mathrm{bp}$ & & & \\
length > 900 bp & & 4 & $0.3 \%$ & \\
length at 800-899 bp & 165 & $14.8 \%$ & $15.1 \%$ \\
length at 700-799 bp & 372 & $33.3 \%$ & $48.4 \%$ \\
length at 600-699 bp & 136 & $12.2 \%$ & $60.6 \%$ \\
length at 500-599 bp & 162 & $14.5 \%$ & $75.1 \%$ \\
length at 400-499 bp & 119 & $10.7 \%$ & $85.8 \%$ \\
length at 300-399 bp & 76 & $6.8 \%$ & $92.6 \%$ \\
length at 200-299 bp & 48 & $4.3 \%$ & $96.9 \%$ \\
length at 100-199 bp & 35 & $3.1 \%$ & $100.0 \%$ \\
\hline Total No. high-quality ESTs & $1,117^{*}$ & & \\
\hline
\end{tabular}

*Raw sequences were automatically trimmed using SeqMan (DNASTAR). After editing only clones above $100 \mathrm{bp}$ in length were retrieved. parameters, $21 \%$ of ESTs did not show significant homology to coding sequences in the GenBank nonredundant database and therefore could not be confidently annotated. BLASTX of the predicted proteins from the longest open reading frame of these unannotated ESTs resulted in the majority of ESTs (75\%) falling below the $10 \mathrm{E}^{-20}$ cut-off value chosen, with the remaining $25 \%$ not showing significant homology to any GenBank proteins. Those undiscovered sequences from $O$. affinis include novel coding sequences, as well as sequences that are non-protein coding. Due the stringent cut-off limit of $10 \mathrm{E}^{-20}$, ESTs with a short length of overlapping open reading frame (ORF) sequence or small ORFs were not annotated.

$79 \%$ of ESTs were annotated by similarity to coding sequences in the GenBank non-redundant database. Of these, approximately $44 \%$ of ESTs shared $80 \%$ or higher identity with existing sequences, $24 \%$ had identity of between $60-80 \%$, and less than $10 \%$ shared less than $60 \%$ identity to known coding sequences. Of these ESTs, $29 \%$ were predicted to be full length cDNA clones, starting with an ATG codon at a similar position to its closest BLASTX hit.

\section{Functional annotation}

ESTs returning a valid BLASTX hit from GenBank were individually searched against The Arabidopsis Information Resource (TAIR) [29] and the Munich Information Center for Protein Sequences (MIPS) MAtDB database [30]. Annotation of $O$. affinis ESTs was based on sequence homology to Arabidopsis genes; ESTs were then functionally annotated according to the MIPS FunCat schema [31].

Of the 880 ESTs returning a valid BLASTX hit, $12.2 \%$ could not be functionally annotated by comparison with genes in Arabidopsis. Cyclotide precursor genes fell within this group since Arabidopsis does not produce cyclotides. $10.7 \%$ of ESTs were found to encode proteins of unknown function. The remaining ESTs were grouped into six main categories (Figure 2): metabolism (28.3\%), information pathway (19.4\%), transport (11.3\%), perception and response to stimuli (12.6\%), developmental processes $(5.2 \%)$ and localisation $(0.3 \%)$. These were further subdivided into 19 subcategories (Table 2) [31].

\section{Contigs composed of most abundant ESTs}

Analysis of EST frequency (abundance) can provide insights into gene expression levels and biochemical functions occurring in a tissue. We identified the 20 most abundantly expressed contigs (Table 3 ). Two contigs of these 20 most abundant ESTs were cyclotide precursor protein genes for kalata B1 (Oak1) and kalata B2 (Oak4), which is consistent with kalata B1 and B2 being the most abundant in cyclotide profiles $[16,17]$. 


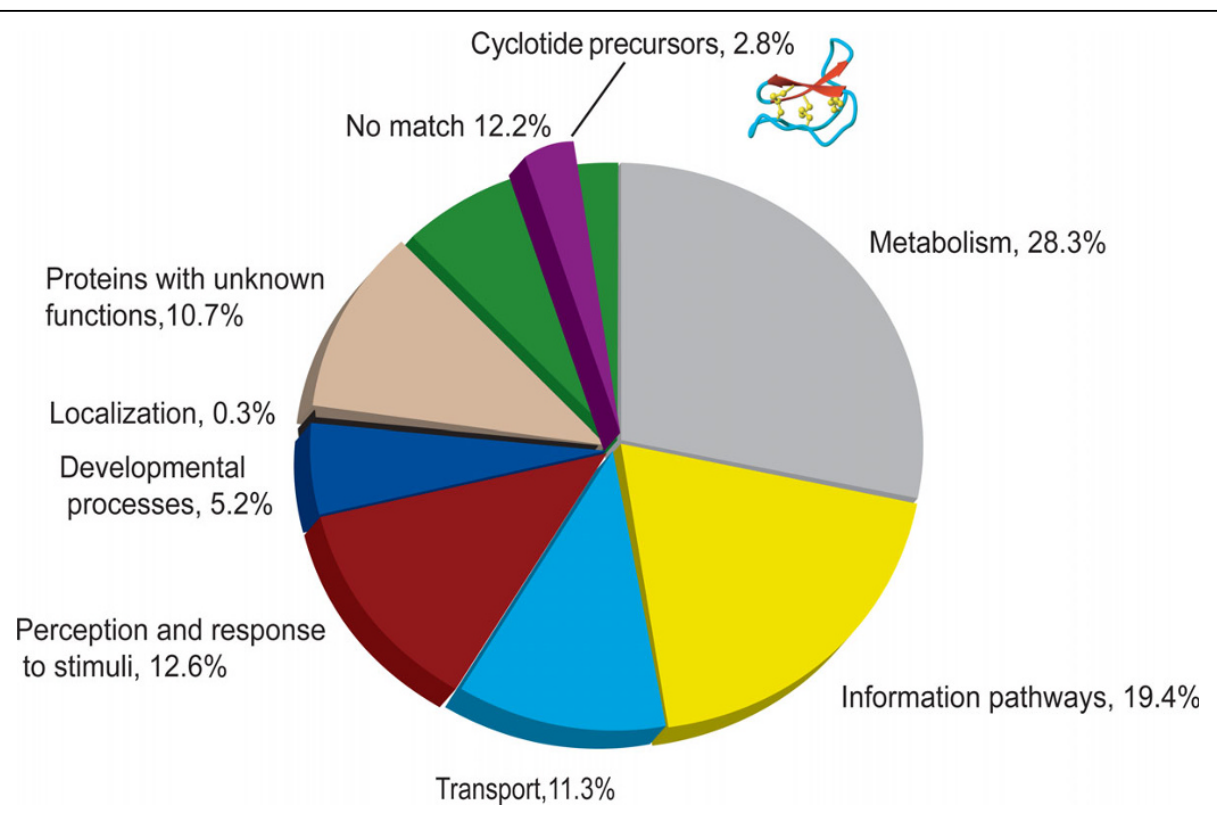

Figure 2 The functional distribution of EST clones by modified MIPS based functional classification for 0 . affinis. BLASTX comparisons to predicted proteins from Arabidopsis were used to assign $O$. affinis ESTs based on functional annotation after MIPS FunCat schema. The cyclotide precursors are pointed out within "No match" group since Arabidopsis does not contain cyclotides.

Table 2 MIPS FunCat analysis of $O$. affinis ESTs compared with Arabidopsis

\begin{tabular}{|c|c|c|}
\hline MIPS Functional category & No. ESTs & $\%$ \\
\hline \multicolumn{3}{|l|}{ Metabolism } \\
\hline 01 metabolism & 196 & $22.3 \%$ \\
\hline 02 energy & 53 & $6.0 \%$ \\
\hline \multicolumn{3}{|l|}{ Information pathways } \\
\hline 10 cell cycle and DNA processing & 7 & $0.8 \%$ \\
\hline 11 transcription & 9 & $1.0 \%$ \\
\hline 12 protein synthesis & 60 & $6.8 \%$ \\
\hline 14 protein fate (folding, modification, destination) & 88 & $10.0 \%$ \\
\hline 16 protein with binding function or cofactor requirement (structural or catalytic) & 7 & $0.8 \%$ \\
\hline \multicolumn{3}{|l|}{ Transport } \\
\hline 20 cellular transport, transport facilities and transport routes & 99 & $11.3 \%$ \\
\hline \multicolumn{3}{|l|}{ Perception and response to stimuli } \\
\hline 30 cellular communication/signal transduction mechanism & 11 & $1.3 \%$ \\
\hline 32 cell rescue, defense and virulence & 74 & $8.4 \%$ \\
\hline 34 interaction with the environment & 25 & $2.8 \%$ \\
\hline 36 systemic interaction with the environment & 1 & $0.1 \%$ \\
\hline \multicolumn{3}{|l|}{ Developmental processes } \\
\hline 40 cell fate & 13 & $1.5 \%$ \\
\hline 41 development (systemic) & 16 & $1.8 \%$ \\
\hline 42 biogenesis of cellular components & 9 & $1.0 \%$ \\
\hline 43 cell type differentiation & 6 & $0.7 \%$ \\
\hline 45 tissue differentiation & 1 & $0.1 \%$ \\
\hline 47 organ differentiation & 1 & $0.1 \%$ \\
\hline \multicolumn{3}{|l|}{ Localisation } \\
\hline 70 subcellular localisation & 3 & $0.3 \%$ \\
\hline Proteins with unknown functions & 94 & $10.7 \%$ \\
\hline No match* & 107 & $12.2 \%$ \\
\hline
\end{tabular}

*The cyclotide precursors are within this subcategory. 
Table 3 The 20 most abundant ESTs isolated from leaf cDNA library of 0 . affinis

\begin{tabular}{llll}
\hline Contig ID & No. ESTs & Putative function & AGI number \\
\hline Oa113 & 21 & 30S ribosomal protein & AT5G24490 \\
Oa126 & 18 & RuBisCO activase & AT2G39730 \\
Oa018 & 14 & undiscovered sequence & AT3G21200 \\
Oa122 & 14 & mitochondrial carnitine Acyl carrier-like protein & AT5G46800 \\
Oa008 & 13 & kalata B2 precursor (Oak4) & \\
Oa014 & 13 & CAAX protease & AT4G01320 \\
Oa131 & 13 & acidic endochitinase (CHIB1) & AT5G24090 \\
Oa031 & 12 & light-dependent NADPH:protochlorophyllide oxidoreductase B & AT4G27440 \\
Oa054 & 12 & fructose-bisphosphate aldolase & AT4G38970 \\
Oa066 & 12 & galacturonosyltransferase & AT5G54690 \\
Oa185 & 12 & glyceraldehyde-3-phosphate dehydrogenase & AT1G13440 \\
Oa064 & 11 & kalata B1 precursor (Oak1) & \\
Oa071 & 11 & S-adenosylmethionine synthetase 3 & AT2G36880 \\
Oa081 & 11 & co-chaperone grpE family protein & AT1G36390 \\
Oa090 & 11 & protein binding & AT1G22970 \\
Oa188 & 11 & asparaginase & AT3G16150 \\
Oa134 & 10 & ankyrin repeat family protein & AT2G01680 \\
Oa115 & 9 & ribosomal protein L1 family protein & AT3G63490 \\
Oa022 & 8 & phosphoribulokinase & AT1G32060 \\
Oa186 & 8 & glyceraldehyde-3-phosphate dehydrogenase & AT1G12900 \\
\hline
\end{tabular}

*The cyclotides precursors are highlighted.

Identification of ESTs involved in cyclotide biosynthesis Although the number of known cyclotide peptide sequences has grown to $>140$ in recent years $[5,32]$, there is still relatively little known about the mechanism by which these peptides are processed in plants. Based on previous work $[18,19]$, we believe that cyclotide precursors enter the secretory pathway where folding and disulfide bond formation occur before cleavage and cyclisation events release the mature cyclic peptides. The presence of putative endoplasmic reticulum (ER) signal sequences in the precursors suggests that cyclotide domains probably fold in the ER where the cysteine residues form three highly conserved disulfide bonds. After correct folding and disulfide bond formation, the precursor protein is processed by cleavage of two peptide bonds and the cyclotide domain is head-to-tail cyclised. Candidate genes for these processes have been identified in this EST sequencing project.

\section{Cyclotide precursor proteins}

The cyclotide precursor proteins of $O$. affinis identified so far include an ER signal sequence, a N-terminal prodomain of variable length and sequences, followed by one to three cyclotide domains (Figure 3) [4,33]. Usually each cyclotide domain ( 28 - 37 residues) is separated by a 25 amino acid region termed the $\mathrm{N}$-terminal repeat (NTR) because it is relatively well conserved within these precursors. Although not part of the mature cyclotide domain, it has been suggested that NTR sequences within the precursors might modulate protein folding [33].

Four cDNA clones encoding cyclotide precursors have been isolated previously from O. affinis. Named Oak1 4, they encode cyclotides kalata B1, B3/B6, B7 and B2 respectively [GenBank: Oak1 AF393825, Oak2 AF393826, Oak3 AF393827, Oak4 AF393828]. We identified 13 ESTs encoding Oak4 (Oa008), 11 encoding Oak1 (Oa064), three ESTs encoding Oak2 (Oa119) and three ESTs encoding Oak3 (Oa070). MALDI-TOF mass spectrometry of leaf peptide extracts showed an abundance of these cyclotides consistent with the frequency of the corresponding ESTs (Figure 4); that is, kalata B2 and kalata B1 are most abundant while kalatas B3, B6 and $B 7$ are less so. This result indicates that cyclotide precursor mRNA expression levels correlate with peptide levels in leaves of $O$. affinis and have not been skewed by amplification of the library by PCR.

Initially 13 ESTs were assembled into contig Oa008 encoding Oak4, the precursor protein for kalata B2, with $90 \%$ minimum match percentage. Re-alignment by ClustalW [34] showed that ESTs within this contig can be divided into two subgroups: namely one subgroup of nine ESTs with $100 \%$ identity to the kalata B2 precursor Oak4 [GenBank: AF393828] and another of four ESTs with $97 \%$ and $95 \%$ similarity to Oak4 at the nucleotide and protein level, respectively. The major differences were found in the 5' untranslated region and at the beginning of the ORF within the ER signal domain. 


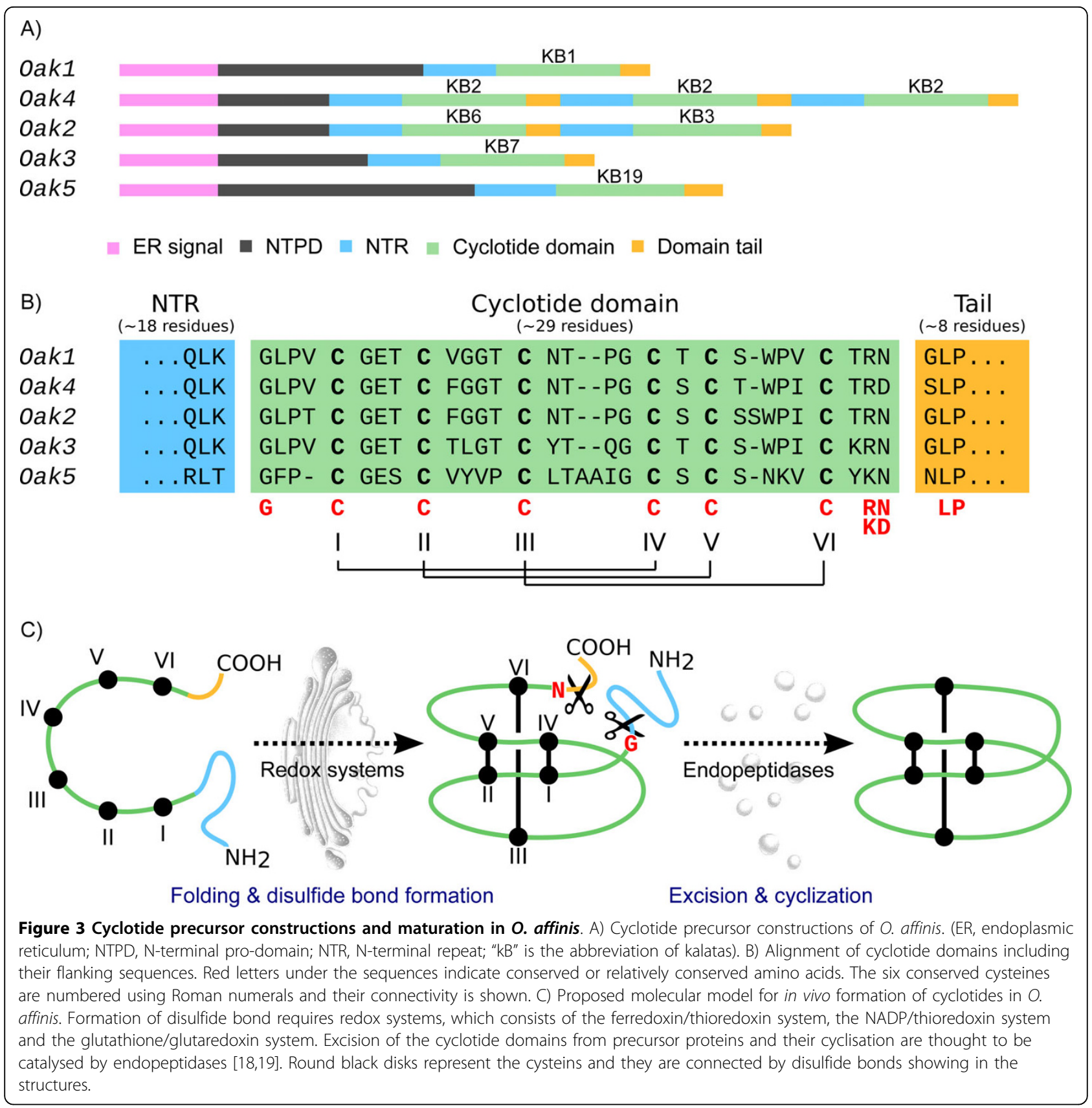

They are most probably variant copies of Oak4, potentially produced by gene duplication.

Previous studies have employed mainly chemical peptide extractions, reverse phase-HPLC and mass spectrometry to isolate cyclotide sequences $[6,35]$. However, only four cDNA sequences for $O$. affinis cyclotide precursors have been determined [4] among the eighteen characterised cyclotides using the approach described above [16]. Because cyclotides precursors from $O$. affinis are only moderately conserved, with sequence identities of approximately $60 \%$, it is difficult to develop a robust strategy for PCR amplification based on sequence similarity. In this EST project, we identified a single EST, contig Oa278, which encodes a previously unidentified putative bracelet cyclotide precursor, which we named Oak5. The cyclotide it encodes was named kalata B19. This is the first bracelet cyclotide precursor cDNA sequence identified in O. affinis; all other known cyclotide gene sequences from $O$. affinis belong to the Möbius sub-class. Möbius sub-class cyclotides contain a conceptual 'twist' (i.e. a cis-X-Pro peptide bond) in their backbone, whereas bracelet cyclotides do not [5]. Oa278 


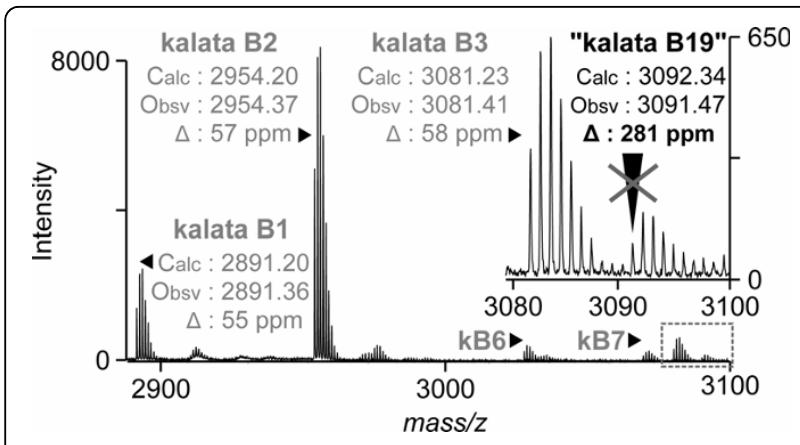

Figure 4 MALDI-TOF MS profile of crude peptide extracts from O. affinis leaves. The calculated (Calc) and observed (Obsv) monoisotopic masses for the cyclotides kalata B1, B2 and B3 are included and serve as internal controls. Peaks for kalata B6 (kB6) and kalata B7 (kB7) are marked without mass data. The calculated mass for kalata B19 (3092.34) is close to a mass in this profile (3091.47), but when its error (represented as ppm) is compared to the errors of other cyclotides in the same profile, the difference between calculated and observed is too great for this mass to be B19.

showed a high degree (80\%) of identity with Leonia cymosa cycloviolin-D [Swiss-Prot: P84640] [36], Viola hederaceae cycloviolacin-H1 [Swiss-Prot: P58433] [5], and Melicytus ramiflorus Mra21, Mra14 and Mra20 [GenBank: ABO21615, ABO21616, ABO21614] [37]. The Asn residue immediately after the mature cyclotide domain in Oak5 is unusual and may affect processing. We confirmed the sequence of the Oak5 EST by independently cloning Oak5 by RT-PCR with specific primers and leaf cDNA.

The sequence of kalata B19 was compared to the characterised cyclotides from O. affinis and it possesses several residues that are conserved across all cyclotides (Table 4), namely six Cys residues and a Gly residue at the N-terminus. Typically, cyclotides contain a conserved Glu in loop 1 (except B12 which contains Asp), Gly in loop 3 (except Ser in B12), and Asn or Asp at the $\mathrm{C}$-terminus. A proline in loop 5 is only conserved in the Möbius subfamily. This conservation still allows for the existence of a great diversity of cyclotide sequences; however it indicates that cyclotide processing for all precursors probably uses the same basic mechanism.

To establish whether Oak5-encoded kalata B19 was detectable in the $O$. affinis cyclotide profile, MALDITOF mass spectrometry was employed. We did not observe a mass consistent with the calculated mass of kB19 in a peptide extract from O. affinis leaves (Figure $3)$. The inability to detect $\mathrm{kB19}$ is the cyclotide profile could be due to Oak 5 mis-processing, but may also be because it falls below the level of detection. Oak5 mRNA is lowly expressed relative to the other Oak genes and if at low levels kB19 may be being masked by other cyclotides.
In total, 31 ESTs encoding putative cyclotide precursors were isolated. This represents $2.8 \%$ of all ESTs sequenced, and a substantial commitment of the $O$. affinis transcriptome to cyclotide biosynthesis. In O. affinis, cyclotides are produced in levels as high as $1-2$ grams per kg wet weight [38], but until now it was uncertain whether this high protein expression correlated with highly abundant mRNA at the level of transcription. Cyclotides have been purified and sequenced from decades-old dried herbarium samples (D. Craik, unpublished), which indicates that cyclotides are remarkably stable in plants. The stability of cyclotides could have meant peptide levels slowly accumulated over the life of the developing plant eventually reaching high levels in adult plants, the stage at which most plants are used for studies. It is interesting to note that mRNA expression levels correlate well with peptide level, suggesting cyclotides might be subject to turnover in planta.

\section{Disulfide formation and oxidative protein folding}

Previous studies show that six cysteine residues in cyclotide precursors form three disulfide bonds [39]. Disulfide bonds are required for the stability and function of a large number of proteins and disulfide bond formation is essential for proper protein folding [40]. There are several systems linking hydrogen donors to an intermediary disulfide protein, namely the ferredoxin/thioredoxin system, the NADP/thioredoxin system and the glutathione/glutaredoxin system; each system acts to effect changes that alter the activity of target proteins. These systems are composed of reduced ferredoxin, thioredoxin, reduced glutathione, glutaredoxin, ferredoxin-thioredoxin reductase, and NADP-thioredoxin reductase [41]. A related disulfide protein, protein disulfide isomerase (PDI) acts in protein assembly [42].

An O. affinis PDI cDNA clone [GenBank: EF611425] was isolated recently and shown to encode a protein that improves in vitro oxidative folding of kalata B1 at physiological $\mathrm{pH}$ [43]. In the current study several other ESTs involved in disulfide bond formation have been identified: Oa408 and Oa412 encoding putative thioredoxins, Oa099 and Oa312 encoding putative glutathione transferases, Oa299 encoding a putative glutaredoxin as well as Oa272 and Oa321 encoding ferredoxins. These enzymes are potential candidates for cyclotide disulfide formation and protein folding in O. affinis.

Thioredoxins are disulfide reductases that promote disulfide bond formation in vivo in the oxidised form, and glutaredoxins are disulfide bond-formation catalysts in E. coli $[44,45]$. The putative thioredoxin Oa408 has $60 \%$ sequence similarity with thioredoxin $m$ from Рopulus trichocarpa [GenBank: EEF06869], wheras Oa412 has 58\% similarity with thioredoxin $x$ from Arabidopsis [GenBank: AAF15952]. Predicted protein products from 
Table 4 Sequences of 0 . affinis cyclotides

\begin{tabular}{|c|c|c|c|c|}
\hline \multicolumn{2}{|c|}{ Möbius } & \multirow{2}{*}{$\begin{array}{c}\operatorname{amino} \\
\text { acids } \\
29\end{array}$} & \multirow{2}{*}{$\begin{array}{c}\text { average mass (Da) } \\
2892.4\end{array}$} & \multirow{2}{*}{$\begin{array}{l}\text { Ref. } \\
\text { Saether et al. [3] }\end{array}$} \\
\hline B1 & G-LPVCGETCVGGTC-NTPGCTCS-WPVCTRN & & & \\
\hline B4 & G-LPVCGETCVGGTC-NTPGCTCS-WPVCTRD & 29 & 2893.3 & Craik et al. [5] \\
\hline B2 & G-LPVCGETCFGGTC-NTPGCSCT-WPICTRD & 29 & 2955.4 & Craik et al. [5] \\
\hline B11 & G-LPVCGETCFGGTC-NTPGCSCT-DPICTRD & 29 & 2884.3 & Plan et al. [16] \\
\hline B15 & G-LPVCGESCFGGSC-YTPGCSCT-WPICTRD & 29 & 2976.4 & Plan et al. [16] \\
\hline B6 & G-LPTCGETCFGGTC-NTPGCSCSSWPICTRN & 30 & 3029.4 & Jennings et al. [4] \\
\hline B10 & G-LPTCGETCFGGTC-NTPGCSCSSWPICTRD & 30 & 3030.4 & Plan et al. [16] \\
\hline B13 & G-LPVCGETCFGGTC-NTPGCACDPWPVCTRD & 30 & 3036.5 & Plan et al. [16] \\
\hline B14 & G-LPVCGESCFGGTC-NTPGCACDPWPVCTRD & 30 & 3022.4 & Plan et al. [16] \\
\hline B3 & G-LPTCGETCFGGTC-NTPGCTCDPWPICTRD & 30 & 3082.5 & Craik et al. [5] \\
\hline B7 & G-LPVCGETCTLGTC-YTQGCTCS-WPICKRN & 29 & 3071.6 & Jennings et al. [4] \\
\hline \multicolumn{5}{|c|}{ Bracelet } \\
\hline B8 & GSVLNCGETCLLGTC-YTTGCTCNKYRVCTKD & 31 & 3283.8 & Daly et al. [55] \\
\hline B9 & GSVFNCGETCVLGTC-YTPGCTCNTYRVCTKD & 31 & 3272.7 & Plan et al. [16] \\
\hline B12 & G-SLCGDTCFVLGC-NDSSCSCN-YPICVKD & 28 & 2880.3 & Plan et al. [16] \\
\hline B16 & G-IPCAESCVYIPCTITALLGCKCQ-DKVCY-D & 30 & 3186.8 & Plan et al. [16] \\
\hline B17 & G-IPCAESCVYIPCTITALLGCKCK-DQVCY-N & 30 & 3185.8 & Plan et al. [16] \\
\hline B18 & G-VPCAESCVYIPC-ISTVLGCSCS-NQVCYRN & 30 & 3145.7 & Seydel et al. [17] \\
\hline B5 & G-TPCGESCVYIPC-ISGVIGCSCT-DKVCYLN & 30 & 3061.6 & Craik et al. [5] \\
\hline B19 & G-FPCGESCVYVPC-LTAAIGCSCS-NKVCYKN & 30 & 3093.7 & this work \\
\hline & * $\quad * .:: * \quad * \quad * * \quad: *$ & & & \\
\hline
\end{tabular}

Multiple sequence alignment of $O$. affinis cyclotides.

both contigs contain features typical of thioredoxins; the conserved thioredoxin catalytic site motif (WCGPC), a characteristic tryptophan residue at the active thiol/disulfide site, and a structural motif thought to be involved in cell-to-cell transfer, which is typical of thioredoxins [46]. Although glutaredoxins function in a similar way to thioredoxins, glutaredoxins have a more positive redox potential $[42,43]$. Oa299, encoding a putative glutaredoxin family protein, shares approximately $40 \%$ sequence identity with other plant glutaredoxins [GenBank: XP_002270142, NP_196885].

A number of other ESTs encoding predicted proteins involved in the regulation of redox environment were identified and included peroxiredoxins (Oa399 and Oa400), and oxidoreductases (Oa031, Oa076, Oa465, Oa476; represented by 16 ESTs). These enzymes typically maintain the redox environment which is crucial for proper disulfide bond formation in vivo [41,47,48], and as such might have a role in the processing of cyclotides.

\section{Excision and ligation of cyclotide domain(s)}

The mechanism of excision and cyclisation that occurs to produce mature cyclotides from their precursor proteins remains unclear. Excision of the cyclotide domain (s) from the precursor and ligation of the newly formed $\mathrm{N}$ - and C-termini probably occurs after folding of the cyclotide domain, but possibly occurs synchronously with formation of the cyclised backbone $[18,19]$.

Although the residues preceding the cyclotide domain are not highly conserved there is a string of conserved sequence flanking the C-terminal Asn/Asp of the mature cyclotide domain. Some insight into the mechanism for carboxyl terminus processing has been gained (Figure 3) $[18,19]$. A residue with a small side chain (Gly, Ala, Ser) usually follows the mature cyclotide domain and itself is followed by an absolutely conserved leucine residue. Asparaginyl endopeptidases (AEPs) are widespread in plants where they are also referred to as vacuolar processing enzymes (VPEs), and specifically cleave peptide bonds on the C-terminal side of Asn and, with less efficiency, Asp [49,50]. The activity of AEP is involved in cyclisation of kalata B1 in tobacco plants transiently transformed with the Oak1 gene [19]. In Oak1 transformed tobacco plants, AEP catalyses protein backbone cyclisation by coupling asparaginyl bond hydrolysis at the $\mathrm{C}$-terminus of the cyclotide domain with peptide bond ligation.

In the current study we identified an AEP homolog from O. affinis [GenBank: EF015631], which shares up to $68 \%$ identity with other plant AEPs [e.g. GenBank: EEF45813, P49043, BAC54827]. The putative AEP from $O$. affinis has the active site "EACES", which is conserved in plant AEPs (E(A/G)CES) [51,52]. A possible 
site for self-cleavage of C-terminal propeptides is present at residue 418 (VDD/W), which is conserved in most of plant AEPs [50]. This putative enzyme belongs to the vegetative-type according to genetic analysis. Since the C-terminus of cyclotide precursors Oak1, Oak2, Oak3 are Asn, followed by Gly, we believe AEPs have an important role in cyclotide domain excision from the precursor proteins. We are currently investigating function of AEPs on the cyclotide hydrolysis and cyclisation in $O$. affinis.

Asparaginases are enzymes that catalyse the hydrolysis of Asn to Asp, releasing $\mathrm{NH}_{4}{ }^{+}$, and have an important role in protein metabolism $[53,54]$. A putative asparaginase, Oa188 comprised of 11 ESTs, is one of the most highly expressed genes in $O$. affinis leaves according to this study (Table 3) and shares up to $86 \%$ identity with other plant asparaginases [GenBank: EEF52347, BAC66615]. There are two pairs of cyclotides in O. affinis that differ from each other by an Asn/Asp change at the C-terminal residue (kalata B6/B10 and kalata B1/ B4). Precursor cDNAs for only one of each pair have been cloned and encode Asn-ending cyclotides, but cDNAs for their Asp-ending counterpart have not yet been identified. The abundance of cDNAs for an enzyme that catalyses a change between Asn and Asp could suggest that these cyclotides are not encoded by different gene products, rather they are produced as a result of post-translational modification.

\section{Conclusions}

In this study we estimated the genome size of $O$. affinis using flow cytometry, and undertook an EST sequencing project on $O$. affinis to identify candidates for in vivo cyclotide biosynthesis and cyclisation. We discovered that the holoploid genome size of $O$. affinis was one of the largest genomes of the Rubiaceae family. 1,117 highquality ESTs were obtained and annotated representing 502 unique transcripts. O. affinis dedicated a substantial portion of its transcriptome to cyclotide production: $2.8 \%$ of ESTs encoded cyclotides. We also discovered a new cDNA encoding an unidentified cyclotide kalata B19 (Oak5), the first cDNA for a bracelet cyclotide in $O$. affinis. As the first sequencing project undertaken in $O$. affinis, this study has uncovered ESTs encoding genes that are potentially involved at each step of cyclotide production and processing, including several cyclotide precursor proteins (Oak1 - 5), folding and disulfide bond formation, regulation of redox environment, and proteolytic processing. Previous attempts to express cyclotides by a transgene in model plants displayed low efficiency and mis-processing; therefore further investigation of the enzymes identified in this study could lead to a deeper understanding of cyclotide processing and improved outcomes for recombinant cyclotide production in plants, which will advance the application of cyclotides for pharmaceutical outcomes and for the protection of crop plants from insect pests.

\section{Methods}

\section{Genome size determination}

Genome size was measured by flow cytometry using Partec CyFlow cytometer (Partec GmbH, Münster, Germany) equipped with a $100 \mathrm{~mW}$ green $(532 \mathrm{~nm})$ solid state laser Cobolt Samba. Sample preparation followed the simplified two-step procedure described by Doležel et al. [23] and Glycine max 'Polanka', 2C DNA $=2.50 \mathrm{pg}$ was used as a suitable internal reference standard. Nuclei from $O$. affinis and the Glycine max 'Polanka' were isolated simultaneously, stained with propidium iodide, and analysed. Three repetitions on different days were performed.

\section{cDNA library construction and EST sequencing}

A leaf cDNA library was constructed [4] and amplified by PCR using universal primers M13 forward and reverse. The resulting PCR products were separated by electrophoresis and fragments larger than 500 bp recovered and cloned into pGEM-T Easy (Invitrogen) for sequencing. The Australian Genome Research Facility Ltd (Brisbane, Australia) randomly selected white colonies, prepared plasmid and sequenced each using the SK primer (5'-CGC TCT AGA ACT AGT GGA TC-3').

\section{EST processing and contig assembly}

Raw EST sequences were automatically trimmed of vector, adapter, and low-quality sequence regions using SeqMan (Lasergene, DNASTAR Inc, Madison, USA). Clones less than $100 \mathrm{bp}$ were eliminated from the data set. All edited EST sequences were submitted to GenBank dbEST. Edited sequences were assembled into contigs or singletons using SeqMan sequence assembly software with the key parameters of minimum $90 \%$ match over 50 bp overlap. Contigs were individually inspected for low-quality sequence, splice variants and chimeras, before ESTs were reassembled.

\section{BLAST analysis and functional annotation}

Analysis of sequences was conducted with the BLASTX server and the National Center for Biotechnology Information non-redundant protein database $[27,28]$. The nearest neighbour hits information was parsed. Returning hits better than the $10 \mathrm{E}^{-20}$ E-value cutoff value imposed were compared with proteins from TAIR [29] and searched against MAtDB to assign functional classification [30]. Functional classification was based on the MIPS FunCat schema [31]. Alignment of ESTs was conducted by Clustal W2 [34] and cyclotide precursors were compared with known sequences in the cyclotide sequence database CyBase [32]. 


\section{Confirmation of novel cyclotide precursor Oak5}

Since Oak5 was represented by only a single EST, we confirmed its sequence by cloning it from O. affinis cDNA. O. affinis leaves were ground to a fine powder under liquid nitrogen and extracted by resuspension of $\sim 0.3 \mathrm{~mL}$ tissue powder in $0.5 \mathrm{~mL}$ of buffer $(0.1 \mathrm{M}$ Tris pH 8.0, 5 mM EDTA, $0.1 \mathrm{M} \mathrm{NaCl}, 0.5 \%$ SDS, $1 \%$ 2-mercaptoethanol), extracting twice with 1:1 phenol:chloroform and precipitating the nucleic acids. Nucleic acid pellets were re-dissolved in $0.5 \mathrm{~mL}$ of water and the RNA selectively precipitated overnight at $4^{\circ} \mathrm{C}$ by addition of lithium chloride to $2 \mathrm{M}$. Total RNA was DNase treated before $1 \mu \mathrm{g}$ was reverse transcribed using Superscript III (Invitrogen). Primers were designed against contig Oa278 which encodes Oak5. PCR was performed using primers JM302 (5'-GAG CTG GGG TGG AGC TTT T-3'), JM303 (5'-TAT TCC AAT TGG GCA ACA AG-3') and the products were cloned into pGEM-Teasy (Promega). Three clones were sequenced and confirmed the Oak5 sequence as correct.

\section{Peptide analysis by MALDI-TOF mass spectrometry}

Leaf extracts were ground in $50 \%(\mathrm{v} / \mathrm{v})$ acetonitrile $(\mathrm{ACN})$ containing $0.05 \%(\mathrm{v} / \mathrm{v})$ trifluoroacetic acid (TFA). Ground tissue was pelleted by centrifugation and the supernatant was freeze-dried and resuspended in $0.05 \%$ TFA. The C18 Ziptip (Millipore) was used to desalt and concentrate the sample. The $\alpha$-cyano-4-hydroxycinnamic acid in $50 \%$ ACN $/ 0.05 \%$ TFA was used as the matrix. Analysis of peptide masses was performed using MALDI-TOF mass spectrometer (Voyager-DE STR) (Applied Biosystems) in positive ion reflector mode with an accelerating voltage of $20,000 \mathrm{~V}$. A 165 ns delay time was used. The data were acquired and processed using the accompanying software. 50 spectra at 20 positions were accumulated per spot. Calibration was conducted using a mixture of peptide standards (MSCal1; SigmaAldrich), and only masses above a signal to noise ratio of 5 - 10 were recorded.

\footnotetext{
Acknowledgements

The authors thank M. Anderson and C. Jennings of La Trobe University for the $O$. affinis CDNA library. We thank J. Botella for help with data analysis, J-C Westermann for comments on the manuscript, C. Wang, M. Colgrave and A. Jones for assistance with mass spectrometry analysis. We thank T. Urfus and $P$. Vít for their help with genome size estimation. This work was supported by grants from the Australian Research Council. Flow cytometric analyses were supported by the Ministry of Education, Youth and Sports of the Czech Republic (project no. MSM 0021620828) and Academy of Sciences of the Czech Republic (no. AV0Z60050516). QQ was supported by the China Scholarship Council and the National Natural Science Foundation of China (No.30771762), QK is an ARC Post-Doctoral Fellow, DC is an NHMRC Principal Research Fellow and JM is an ARC QEll Fellow.
}

\section{Author details}

${ }^{1} T$ The University of Queensland, Institute for Molecular Bioscience, Brisbane, Queensland, 4072, Australia. ${ }^{2}$ School of Agriculture and Food Science,
Zhejiang Forestry University, Hangzhou, Zhejiang, 311300, China. ${ }^{3}$ Department of Botany, Faculty of Science, Charles University, Benatska 2, Prague, 128 01, Czech Republic. ${ }^{4}$ Institute of Botany, Academy of Sciences, Pruhonice 1, 252 43, Czech Republic. ${ }^{5}$ Current address: Institut de Biologie Moléculaire des Plantes du CNRS, Université de Strasbourg, 12 rue du Général Zimmer, 67084 Strasbourg Cedex, France. ${ }^{6}$ Current address: Plant Immunity Research Team, RIKEN Plant Science Center, Tsurumi-ku, Yokohama, Japan.

\section{Authors' contributions}

All authors were involved with experiments and data collection. DC and JM planned the project. QQ, JM, QK and EM designed and performed experiments. QQ and QK annotated the ESTs. IS performed some gene characterisation. JS performed the genome size determination. QQ, EM, DC and JM wrote the manuscript and all authors read and approved the final manuscript.

Received: 30 November 2009

Accepted: 16 February 2010 Published: 16 February 2010

\section{References}

1. Gran L: An oxytocic principle found in Oldenlandia affinis DC. Medd Nor Farm Selsk 1970, 12:173-180.

2. Sletten K, Gran L: Some molecular properties of kalatapeptide B-1. A uterotonic polypeptide isolated from Oldenlandia affinis DC. Medd Nor Farm Selsk 1973, 7-8:69-82.

3. Saether O, Craik DJ, Campbell ID, Sletten K, Juul J, Norman DG: Elucidation of the primary and three-dimensional structure of the uterotonic polypeptide kalata B1. Biochemistry 1995, 34:4147-4158.

4. Jennings C, West J, Waine C, Craik D, Anderson M: Biosynthesis and insecticidal properties of plant cyclotides: the cyclic knotted proteins from Oldenlandia affinis. Proc Natl Acad Sci USA 2001, 98:10614-10619.

5. Craik DJ, Daly NL, Bond T, Waine C: Plant cyclotides: A unique family of cyclic and knotted proteins that defines the cyclic cystine knot structural motif. J Mol Biol 1999, 294:1327-1336.

6. Gruber CW, Elliott AG, Ireland DC, Delprete PG, Dessein S, Göransson U, Trabi M, Wang CK, Kinghorn AB, Robbrecht E, Craik DJ: Distribution and evolution of circular miniproteins in flowering plants. Plant Cell 2008, 20:2471-2483.

7. Colgrave ML, Craik DJ: Thermal, chemical, and enzymatic stability of the cyclotide kalata B1: the importance of the cyclic cystine knot. Biochemistry 2004, 43:5965-5975.

8. Barbeta BL, Marshall AT, Gillon AD, Craik DJ, Anderson MA: Plant cyclotides disrupt epithelial cells in the midgut of lepidopteran larvae. Proc Natl Acad Sci USA 2008, 105:1221-1225.

9. Jennings $\mathrm{CV}$, Rosengren $\mathrm{KJ}$, Daly NL, Plan M, Stevens J, Scanlon MJ, Waine C, Norman DG, Anderson MA, Craik DJ: Isolation, solution structure, and insecticidal activity of kalata B2, a circular protein with a twist: do Mobius strips exist in nature?. Biochemistry 2005, 44:851-860.

10. Craik DJ, Cemazar M, Daly NL: The cyclotides and related macrocyclic peptides as scaffolds in drug design. Curr Opin Drug Discov Devel 2006, 9:251-260.

11. Craik DJ, Daly NL, Mulvenna J, Plan MR, Trabi M: Discovery, structure and biological activities of the cyclotides. Curr Prot Pept Sci 2004, 5:297-315.

12. Colgrave ML, Kotze AC, Huang YH, O'Grady J, Simonsen SM, Craik DJ: Cyclotides: natural, circular plant peptides that possess significant activity against gastrointestinal nematode parasites of sheep. Biochemistry 2008, 47:5581-5589.

13. Colgrave ML, Kotze AC, Ireland DC, Wang CK, Craik DJ: The anthelmintic activity of the cyclotides: natural variants with enhanced activity. Chembiochem 2008, 9:1939-1945.

14. Colgrave ML, Kotze AC, Kopp S, McCarthy JS, Coleman GT, Craik DJ: Anthelmintic activity of cyclotides: In vitro studies with canine and human hookworms. Acta Trop 2009, 109:163-166.

15. Gran L, Sandberg F, Sletten K: Oldenlandia affinis (R\&S) DC. A plant containing uteroactive peptides used in African traditional medicine. $J$ Ethnopharmacol 2000, 70:197-203.

16. Plan MRR, Göransson U, Clark RJ, Daly NL, Colgrave ML, Craik DJ: The cyclotide fingerprint in Oldenlandia affinis: elucidation of chemically modified, linear and novel macrocyclic peptides. Chembiochem 2007, 8:1001-1011. 
17. Seydel P, Gruber C, Craik D, Dörnenburg H: Formation of cyclotides and variations in cyclotide expression in Oldenlandia affinis suspension cultures. Appl Microbiol Biotechnol 2007, 77:275-284.

18. Gillon AD, Saska I, Jennings CV, Guarino RF, Craik DJ, Anderson MA: Biosynthesis of circular proteins in plants. Plant J 2008, 53:505-515.

19. Saska I, Gillon AD, Hatsugai N, Dietzgen RG, Hara-Nishimura I, Anderson MA, Craik DJ: An asparaginyl endopeptidase mediates in vivo protein backbone cyclisation. J Biol Chem 2007, 282:29721-29728.

20. Lange BM, Wildung MR, Stauber EJ, Sanchez C, Pouchnik D, Croteau R: Probing essential oil biosynthesis and secretion by functional evaluation of expressed sequence tags from mint glandular trichomes. Proc Natl Acad Sci USA 2000, 97:2934-2939.

21. Davis EM, Ringer KL, McConkey ME, Croteau R: Monoterpene metabolism. Cloning, expression, and characterization of menthone reductases from peppermint. Plant Physiol 2005, 137:873-881.

22. Newcomb RD, Crowhurst RN, Gleave AP, Rikkerink EH, Allan AC, Beuning LL, Bowen JH, Gera E, Jamieson KR, Janssen BJ, Laing WA, McArtney S, Nain B, Ross GS, Snowden KC, Souleyre EJ, Walton EF, Yauk YK: Analyses of expressed sequence tags from apple. Plant Physiol 2006, 141:147-166.

23. Doležel J, Greilhuber J, Suda J: Estimation of nuclear DNA content in plants using flow cytometry. Nat Protoc 2007, 2:2233-2244.

24. Udall JA, Swanson JM, Haller K, Rapp RA, Sparks ME, Hatfield J, Yu Y, Wu Y, Dowd C, Arpat AB, Sickler BA, Wilkins TA, Guo JY, Chen XY, Scheffler J, Taliercio E, Turley R, McFadden H, Payton P, Klueva N, Allen R, Zhang D, Haigler C, Wilkerson C, Suo J, Schulze SR, Pierce ML, Essenberg M, Kim H, Llewellyn DJ, Dennis ES, Kudrna D, Wing R, Paterson AH, Soderlund C, Wendel JF: A global assembly of cotton ESTs. Genome Res 2006, 16:441-450.

25. Ramirez M, Graham MA, Blanco-Lopez L, Silvente S, Medrano-Soto A, Blair MW, Hernandez G, Vance CP, Lara M: Sequencing and analysis of common bean ESTs. Building a foundation for functional genomics. Plant Physiol 2005, 137:1211-1227.

26. Moyle R, Fairbairn DJ, Ripi J, Crowe M, Botella JR: Developing pineapple fruit has a small transcriptome dominated by metallothionein. J Exp Bot 2005, 56:101-112.

27. Altschul SF, Madden TL, Schaffer AA, Zhang J, Zhang Z, Miller W, Lipman DJ: Gapped BLAST and PSI-BLAST: a new generation of protein database search programs. Nucleic Acids Res 1997, 25:3389-3402.

28. Wheeler DL, Church DM, Federhen S, Lash AE, Madden TL, Pontius JU, Schuler GD, Schriml LM, Sequeira E, Tatusova TA, Wagner L: Database resources of the National Center for Biotechnology. Nucleic Acids Res 2003, 31:28-33.

29. Rhee SY, Beavis W, Berardini TZ, Chen G, Dixon D, Doyle A, GarciaHernandez M, Huala E, Lander G, Montoya M, Miller N, Mueller LA, Mundodi S, Reiser L, Tacklind J, Weems DC, Wu Y, Xu I, Yoo D, Yoon J, Zhang P: The Arabidopsis Information Resource (TAIR): a model organism database providing a centralized, curated gateway to Arabidopsis biology, research materials and community. Nucleic Acids Res 2003, 31:224-228.

30. Schoof H, Zaccaria P, Gundlach H, Lemcke K, Rudd S, Kolesov G, Arnold R, Mewes HW, Mayer KF: MIPS Arabidopsis thaliana Database (MAtDB): an integrated biological knowledge resource based on the first complete plant genome. Nucleic Acids Res 2002, 30:91-93.

31. Ruepp A, Zollner A, Maier D, Albermann K, Hani J, Mokrejs M, Tetko I, Guldener U, Mannhaupt G, Munsterkotter M, Mewes HW: The FunCat, a functional annotation scheme for systematic classification of proteins from whole genomes. Nucleic Acids Res 2004, 32:5539-5545.

32. Wang CKL, Kaas Q, Chiche L, Craik DJ: CyBase: a database of cyclic protein sequences and structures, with applications in protein discovery and engineering. Nucleic Acids Res 2008, 36:D206-210.

33. Dutton JL, Renda RF, Waine C, Clark RJ, Daly NL, Jennings CV, Anderson MA, Craik DJ: Conserved structural and sequence elements implicated in the processing of gene-encoded circular proteins. $J$ Bio Chem 2004, 279:46858-46867.

34. Larkin MA, Blackshields G, Brown NP, Chenna R, McGettigan PA, McWilliam H, Valentin F, Wallace IM, Wilm A, Lopez R, Thompson JD, Gibson TJ, Higgins DG: Clustal W and Clustal X version 2.0. Bioinformatics 2007, 23:2947-2948.

35. Claeson P, Göransson U, Johansson S, Luijendijk T, Bohlin L: Fractionation protocol for the isolation of polypeptides from plant biomass. J Nat Prod 1998, 61:77-81.
36. Hallock YF, Sowder RCI, Pannell LK, Hughes CB, Johnson DG, Gulakowski R, Cardellina JHI, Boyd MR: Cycloviolins A-D, anti-HIV macrocyclic peptides from Leonia cymosa. J Org Chem 2000, 65:124-128.

37. Trabi M, Mylne JS, Sando L, Craik DJ: Circular proteins from Melicytus (Violaceae) refine the conserved protein and gene architecture of cyclotides. Org Biomol Chem 2009, 7:2378-2388.

38. Gran L: Oxytocic principles of Oldenlandia affinis. Lloydia 1973, 36:174-178.

39. Trabi M, Craik DJ: Circular proteins - no end in sight. Trends Biochem Sci 2002, 27:132-138.

40. Wedemeyer WJ, Welker E, Narayan M, Scheraga HA: Disulfide bonds and protein folding. Biochemistry 2000, 39:4207-4216.

41. Buchanan $B B$, Balmer Y: Redox regulation: a broadening horizon. Annu Rev Plant Biol 2005, 56:187-220.

42. Tu BP, Weissman JS: Oxidative protein folding in eukaryotes: mechanisms and consequences. J Cell Biol 2004, 164:341-346.

43. Gruber CW, Cemazar M, Clark RJ, Horibe T, Renda RF, Anderson MA, Craik DJ: A novel plant protein-disulfide isomerase involved in the oxidative folding of cystine knot defense proteins. J Biol Chem 2007, 282:20435-20446.

44. Debarbieux L, Beckwith J: The reductive enzyme thioredoxin 1 acts as an oxidant when it is exported to the Escherichia coli periplasm. Proc Natl Acad Sci USA 1998, 95:10751-10756.

45. Eser M, Masip L, Kadokura H, Georgiou G, Beckwith J: Disulfide bond formation by exported glutaredoxin indicates glutathione's presence in the E. coli periplasm. Proc Natl Acad Sci USA 2009, 106:1572-1577.

46. Gelhaye E, Rouhier N, Navrot N, Jacquot JP: The plant thioredoxin system. Cell Mol Life Sci 2005, 62:24-35.

47. Dietz KJ: Plant peroxiredoxins. Annu Rev Plant Biol 2003, 54:93-107.

48. König J, Baier M, Horling F, Kahmann U, Harris G, Schürmann P, Dietz K-J: The plant-specific function of 2-Cys peroxiredoxin-mediated detoxification of peroxides in the redox-hierarchy of photosynthetic electron flux. Proc Natl Acad Sci USA 2002, 99:5738-5743.

49. Hatsugai N, Kuroyanagi M, Yamada K, Meshi T, Tsuda S, Kondo M, Nishimura M, Hara-Nishimura I: A plant vacuolar protease, VPE, mediates virus-induced hypersensitive cell death. Science 2004, 305:855-858.

50. Shimada T, Yamada K, Kataoka M, Nakaune S, Koumoto Y, Kuroyanagi M, Tabata S, Kato T, Shinozaki K, Seki M, Kobayashi M, Kondo M, Nishimura M, Hara-Nishimura I: Vacuolar processing enzymes are essential for proper processing of seed storage proteins in Arabidopsis thaliana. I Biol Chem 2003, 278:32292-32299.

51. Hara-Nishimura I, Hatsugai N, Nakaune S, Kuroyanagi M, Nishimura M: Vacuolar processing enzyme: an executor of plant cell death. Curr Opin Plant Biol 2005, 8:404-408.

52. Yamada K, Shimada T, Nishimura M, Hara-Nishimura I: A VPE family supporting various vacuolar functions in plants. Physiol Plant 2005, 123:369-375.

53. Bruneau L, Chapman R, Marsolais F: Co-occurrence of both L-asparaginase subtypes in Arabidopsis: At3g16150 encodes a K+-dependent Lasparaginase. Planta 2006, 224:668-679.

54. Sieciechowicz KA, Joy KW, Ireland RJ: The metabolism of asparagine in plants. Phytochemistry 1988, 27:663-671.

55. Daly NL, Clark RJ, Plan MR, Craik DJ: Kalata B8, a novel antiviral circular protein, exhibits conformational flexibility in the cystine knot motif. Biochem J 2006, 393:619-626.

doi:10.1186/1471-2164-11-111

Cite this article as: Qin et al.: Identification of candidates for cyclotide biosynthesis and cyclisation by expressed sequence tag analysis of Oldenlandia affinis. BMC Genomics 2010 11:111. 